Aim Update our systematic review of probiotic supplementation (Started within first 10 days, duration: 37 days) in preventing 3 stage II NEC in preterm VLBW neonates.

Method Standard Cochrane Neonatal Review Group search strategy was followed. CENTRAL, MEDLINE, EMBASE, CINAHL databases, proceedings (from 2009) of the Pediatric Academic Society meetings and Gastroenterology conferences were searched in September 2011

Results Total 17 RCTs ( $\mathrm{N}=3147$ ) including rrecently published 5 new RCTs $(\mathrm{N}=840)$ were eligible for inclusion in the meta-analysis. The risk of NEC [RR: 0.39; 95\% CI: 0.27, 0.56; p<0.00001] and death (RR: 0.54; 95\% CI: 0.41, 0.72) p<0.0001] was significantly lower and the time to full feeds was significantly shorter in the probiotic group $(\mathrm{WMD}=-2.29$ days; $95 \% \mathrm{CI}:-4.25,-0.32 ; \mathrm{p}<0.00001)$. Risk of sepsis was similar in both groups (RR: 0.92; 95\% CI: 0.80, 1.06). Subgroup analysis, according to baseline incidence of NEC ( $<6 \%$ and $\geq 6 \%$ ) also showed significant benefits of probiotics in both scenarios.

Conclusions The results of our updated Meta analysis continue to show the benefits of probiotic supplementation. We have now provided additional evidence of its benefits in units with high as well as low baseline incidence of NEC.

References (1.2): Deshpande et al, Lancet 2007 and Pediatrics 2010 (3): Alfaleh et al 2011.

\section{NECROTIZING ENTEROCOLITIS IN MULTIPLE GESTATIONS: COMPARISON WITH SINGLETONS}

doi:10.1136/archdischild-2012-302724.1377

${ }^{1} \mathrm{~N}$ Zampieri, ${ }^{2} \mathrm{C}$ Zamboni, 'G Scirè, ${ }^{1} \mathrm{~A}$ Mantovani, $1 \mathrm{~F}$ Camoglio. 'Department of Anesthetic and Surgical Sciences, Pediatric Surgical Unit, University of Verona, Policlincio G.B.Rossi; ${ }^{2}$ Department of Obstetrics and Gynecology, Prenatal Diagnsosi Unit, Civile Maggiore Hospital, Verona, Italy

NEC is one of the most important surgical disease in the first few days after birth. The aim of this study is to describe incidence of Necrotizing enterocolitis in multiple gestations compared with singletons, determining the neonatal outcome, risk factors and comorbid factors.

A retrospective review of the discharge records of multiple-gestation and singletons infants admitted into the neonatal intensive care units between January 2002 and January 2009 was performed.

The medical charts of all infants developing NEC or suspected NEC were reviewed and perinatal data recorded. The risk and comorbid factors of two main groups (developing NEC and not developing NEC) were analyzed.

During the study period we considered 409 infants from multiple gestations and 895 singletons. The percentage of infants with NEC in multiple gestation (18\%) was higher than singleton prematurity at the same Hospital $(4 \%)(p<0.05)$. Patients with suspected or advanced NEC showed longer time of meconium evacuation if compared to the others (mean 5 vs. 2 days, $\mathrm{p}<0.05$ ). Patients who received bowel enemas starting from day 2 after birth did not developed NEC or advanced NEC $(p<0.05)$. Mortality was associated with lower gestational age and lower Apgar score at 1 minute $(\mathrm{p}<0.05)$.

The analysis of multiple pregnancies showed that the incidence of NEC(stage II but Stage III) increased with respect to singleton pregnancies only when considered in relation to a higher prematurity rate.

\section{MECONIUM OBSTRUCTION IN VERY-LOW-BIRTH-WEIGHT PREMATURE INFANTS}

doi:10.1136/archdischild-2012-302724.1378

'E Okulu, 'D Yıldız, 'S Alan, 'A Kilıc, 'IM Akin, 'B Atasay, 'S Arsan, 'M Koloğlu, ${ }^{2} \mathrm{~A}$ Yağ murlu, ${ }^{2} \mathrm{H}$ Dindar. ${ }^{2}$ Department of Pediatrics, Division of Neonatology; ${ }^{2}$ Depertment of Pediatric Surgery, Ankara University, Ankara, Turkey
Aim Meconium obstruction( $\mathrm{MO})$ is a clinical entity primarily affecting very-low-birth-weight(VLBW) premature infants. Intestinal obstruction symptoms in a VLBW infant who did not have evidence of necrotizing enterocolitis, did not have another mechanical or functional cause of obstruction defined as $\mathrm{MO}$.

Method We report a series of 14 infants, whose clinical course is indicative of $\mathrm{MO}$ of prematurity.

Results One-hundred-thirty VLBW infants born between January 1, 2010 and December 31, 2011. Fourteen(11\%) patients were diagnosed as $\mathrm{MO}$. Mean gestational age and birth weight were $28.8 \pm 2.6$ weeks and $943.7 \pm 238.3$ g, respectively. Ninety-three percent of infants were delivered by C/S. Eight (57\%) of infants had afermentioned prenatal risk factors for $\mathrm{MO}$. The time of the passage of the first meconium was between 10-to-72 hours. All patients presented with distended abdomen and feeding intolerance. Abdominal plain x-rays showed multiple distended intestinal loops without air-fluid levels in all cases. Medical therapy, consisting of rectal enema with saline, if failed, enema with $\mathrm{N}$-acetylcysteine was performed. Eleven of the patients underwent ileostomy surgery between posnatal age of 2-to-43 days. Nine patients (64\%) survived, and the times to full enteral feeding was between day of life 13-to-81 days, the median length of hospital stay was 50 days (range 15-92 days) in these patients. One of the patients was diagnosed as Hirschsprung's disease in the follow-up period.

Conclusion VLBW infants with MO can be diagnosed based on their typical clinical and plain radiographic characteristics. Medical management is effective, whereas some cases may need surgically management as ours.

\section{TRANSFUSION-ASSOCIATED NECROTIZING ENTEROCOLITIS IN VERY LOW BIRTH WEIGHT PREMATURE INFANTS}

doi:10.1136/archdischild-2012-302724.1379

${ }^{1} \mathrm{G}$ Demirel, ' ${ }^{1} \mathrm{H}$ Celik, ${ }^{1} \mathrm{HT}$ Aksoy, ${ }^{10} \mathrm{C}$ Erdeve, 'SS Oguz, 'N Uras, 1,2U Dilmen ${ }^{1}$ Neonatology, Zekai Tahir Burak Maternity and Teaching Hospital; ${ }^{2}$ Pediatrics, Yildirim Beyazit University, Faculty of Medicine, Ankara, Turkey

Background and Aims Our aim was to determine the relationship between red blood cell transfusion and necrotizing enterocolitis (NEC) in all admitted very low birth weight (VLBW) infants with or without transfusion.

Methods All VLBW neonates were categorized into five groups:

1. subjects that developed NEC $<48$ hours after transfusion $(n=15)$;

2. subjects that developed NEC $>48$ hours after transfusion $(n=31)$

3. subjects that were never transfused but developed NEC, $(n=50)$;

4. subjects that were transfused but did not develop NEC, $(\mathrm{n}=250)$;

5. and subjects that were neither transfused nor developed $\operatorname{NEC}(n=301)$

Results A group of 647 infants were enrolled in the study. Mean gestational age and birth weight of the patients were $29 \pm 3.1$ weeks and $1157 \pm 237$ grams, respectively. The mean age at the onset of NEC in the NEC groups were $20 \pm 2.3$ days, $12 \pm 3$ days, and $11 \pm 2.6$ days, respectively $(\mathrm{P}<0.05)$. The mean interval from the last transfusion to the onset of NEC was $16.8 \pm 8.8$ hours in Group 1 and $240 \pm 50$ hours in Group $2(\mathrm{p}<0.05)$.

Conclusion In this study, we sought to evaluate all VLBW infants, whether they received a transfusion or not. We suggest that transfusion associated NEC exists, but many other factors influence this multifactorial disease. The age of NEC onset was later in transfused versus non-transfused patients, whereas the interval between transfusion and NEC was shorter. 


\section{A NETWORK PERSPECTIVE OF NECROTISING ENTEROCOLITIS REQUIRING SURGERY}

doi:10.1136/archdischild-2012-302724.1380

M Nash, M Passant, V Rasiah. South West Midlands Neonatal Network, Birmingham, UK

Introduction Necrotising-enterocolitis is the commonest gastrointestinal emergency in very low birth weight (VLBW) infants. The incidence is around 7-per-100 births of VLBW infants. Confirmed cases require aggressive medical therapy, nil-by-mouth for 7-14 days and triple antibiotic therapy. Timely surgical intervention is necessary in those who perforate or are critically ill.

Aim To give a network perspective on the diagnosis, management and outcome of infants affected by NEC focusing on those requiring surgery.

Method Data was retrospectively collected from the Badger database over two years for babies within the South-West Midlands Newborn-Network with a diagnosis of NEC. Babies who were treated for at least 7 days were included, focusing on those requiring surgery.

Results

Abstract 1380 Table 1

\begin{tabular}{ccccc}
\hline & Distinct admissions & $<\mathbf{1 5 0 0 g}$ & Diagnosis NEC & NBM $>$ 7days \\
\hline Number & 8750 & 1407 & 266 & 161 \\
\hline
\end{tabular}

Abstract 1380 Table 2 Outcome

\begin{tabular}{lcccc}
\hline & Number & Survived to discharge & Died & Mortality \\
\hline Surgical & 56 & 48 & 8 & $14 \%$ \\
Conservative & 105 & 88 & 17 & $16 \%$ \\
Overall & 161 & 136 & 25 & $15 \%$ \\
\hline
\end{tabular}

Discussion The standardised use of Badger information system across the network allowed us to audit the outcome of babies with NEC within our network. This is limited by the inter-user variation in quality and detail of information entered.

The National Neonatal Collaborative Necrotizing Enterocolitis Study is currently underway. Our audit provides some insight into the limitations of using Badger alone as a source of information. We need to improve the quality of data entered into Badger.

\section{GASTROINTESTINAL PERFORATIONS IN NEONATAL PERIOD}

doi:10.1136/archdischild-2012-302724.1381

${ }^{1} \mathrm{E}$ Blevrakis, 'C Seremeti, 'N Partalis, ${ }^{1} \mathrm{O}$ Dede, ${ }^{2} \mathrm{~A}$ Alegakis, ${ }^{3} \mathrm{E}$ Korakaki, ${ }^{3} \mathrm{C}$ Gianakopoulou, ${ }^{1} \mathrm{G}$ Sakellaris. 'Department of Paediatric Surgery, University Hospital of Heraklion, Crete; ${ }^{2}$ Department of Toxicology, University of Crete; ${ }^{3}$ Neonatal Intensive Care Unit, University Hospital of Heraklion, Crete, Heraklion, Greece

Aim This report describes our experience concerning gastrointestinal perforation (GI) due to Necrotizing enterocolitis (NEC) during a 10 year period.

Method The cases of 27 GI perforations, which were treated in our hospital, were retrospectively reviewed.

Results All patients were neonates and infants up to the age of 2 months. The study population consisted of 16 boys (59.3\%) and 11 girls $(40.7 \%)$. Twenty one $(77.8 \%)$ neonates were pre-term and the median gestational age was 28 weeks. Twenty four (88.9\%) cases of perforation underwent laparotomy. The overall mortality was $63 \%$. Seventy six percent of the pre-term neonates and only $16.7 \%$ of the full-term died.

Conclusions GI perforation is still connected with a high mortality rate, with NEC being the main cause of death. The neonates who did not undergo surgery all died.

\section{INTRODUCING ROUTINE PROBIOTICS FOR PRETERM NEONATES - NEPEAN HOSPITAL SYDNEY AUSTRALIA APPROACH}

doi:10.1136/archdischild-2012-302724.1382

1,2G Deshpande, 1,2V Shingde, 1,2L Downe, 2,3M Leroi, ' J Xiao. 'Neonatology, Nepean Hospital Sydney; ${ }^{2}$ Nepean Clinical School, University of Sydney, Kingswood, NSW, Australia; ${ }^{3}$ Microbiology, Nepean Hospital Sydney, Kingswood, Austria

Background Probiotic supplementation significantly reduces the risk of mortality and $\geq$ Stage II necrotising enterocolitis in preterm neonates. (Deshpande et al Lancet 2007, Pediatrics 2010). Based on the current evidence, experts favour routine probiotic supplementation in preterm neonates, if safe and effective products are available. We implemented a stepwise plan to introduce probiotics in our regional tertiary neonatal centre.

Step wise approach:

1. Comprehensive literature review to develop evidence based guidelines for optimal use of probiotics in preterm neonates.

2. Identifying a suitable product based on the systematic review of RCTs in preterm neonates, and probiotics listed by Therapeutic Goods Administration, (TGA).

3. Approval of selected probiotic product (Infloran) from local area drug and therapeutics committee.

4. Endorsement from local Ethics Committee.

5. Authorised Prescriber application to TGA via Pharmacy Department.

6. Approval from authorities regarding importing probiotic product from overseas.

7. Independent quality assessment (Taxonomy confirmation, antibiotic susceptibility, and osmolarity).

8. Further assessment for ruling out contamination from a TGA approved laboratory.

Results 2/19 locally available products were potentially appropriate for use in preterm neonates but turned out suboptimal in quality despite manufacturer's data. (Patole et al PSANZ 2010) An overseas proven probiotic product was selected (Infloran) and imported after following above stepwise approach. After vigorous quality assessment Infloran was introduced routinely in preterm neonates at Nepean Hospital Sydney.

Conclusion Safe and effective probiotic product is accessible in Australia for routine use in preterm neonates. Further co-operation is needed between industry, regulatory authorities and clinicians to simplify this process.

\section{IMPROVEMENT OF GUT MICROBIOTA AFTER PROBIOTIC TREATMENT OF PREMATURE NEONATES FOR THE PREVENTING OF NECROTIZING ENTEROCOLITIS}

doi:10.1136/archdischild-2012-302724.1383

\section{B Smith. Statens Serum Institut, Copenhagen, Denmark}

Purpose Necrotizing enterocolitis (NEC) is the most common acquired disease of the gastrointestinal tract in premature neonates. Probiotic supplementation with Lactobacillus rhamnosus and Bifidobacterium lactis was introduced to reduce NEC and potentially provide benefits for the preterm neonates. We study if premature neonates were colonized with $L$. rhamnosus and B. lactis and investigated what influence probiotics had on clinical outcomes.

Methods Faecal samples and clinical information from neonates before and after the introduction of probiotic were collected. 254 premature neonates without probiotics and 101 premature neonates with probiotics all less than 30 weeks of gestation were enrolled. Fecal samples and clinical information during the first month of life from all premature neonates were collected.

Results Clinical information on all premature neonate regarding, gestational age, birth weight, probiotic, antibiotics, food, NEC and dead were collected. A total of 461 feacal samples from premature 